\title{
Understanding magnetic relaxation in single-ion magnets with high blocking temperature
}

\author{
A. Chiesa $\odot,{ }^{1,2}$ F. Cugini $\odot,{ }^{1}$ R. Hussain,,${ }^{1,3}$ E. Macaluso, ${ }^{1}$ G. Allodi, ${ }^{1}$ E. Garlatti,,${ }^{1,2}$ M. Giansiracusa, ${ }^{4}$ C. A. P. Goodwin, \\ F. Ortu, ${ }^{4}$ D. Reta, ${ }^{4}$ J. M. Skelton $\odot,{ }^{4}$ T. Guidi $\odot,{ }^{5}$ P. Santini, ${ }^{1,2}$ M. Solzi $\odot,{ }^{1}$ R. De Renzi, ${ }^{1}$ D. P. Mills $\odot,{ }^{4, *}$ \\ N. F. Chilton $\odot,{ }^{4, \dagger}$ and S. Carretta $\oplus^{1,2, \dagger}$ \\ ${ }^{1}$ Dipartimento di Scienze Matematiche, Fisiche e Informatiche, Università di Parma, I-43124 Parma, Italy \\ ${ }^{2}$ UdR Parma, INSTM, I-43124 Parma, Italy \\ ${ }^{3}$ Dipartimento di Fisica, Università di Pavia, Pavia, Italy \\ ${ }^{4}$ Department of Chemistry, The University of Manchester, Oxford Road, Manchester M13 9PL, United Kingdom \\ ${ }^{5}$ ISIS Facility, Rutherford Appleton Laboratory, OX11 0QX Didcot, United Kingdom
}

(Received 28 November 2019; accepted 16 March 2020; published 4 May 2020)

\begin{abstract}
The recent discovery of single-ion magnets with magnetic hysteresis above liquid-nitrogen temperatures placed these compounds among the best candidates to realize high-density storage devices. Starting from a prototypical dysprosocenium molecule, showing hysteresis up to $60 \mathrm{~K}$, we derive here a general recipe to design high-blocking-temperature rare-earth single-ion magnets. The complex magnetic relaxation is unraveled by combining magnetization and nuclear magnetic resonance measurements with inelastic neutron scattering experiments and $a b$ initio calculations, thus disentangling the different mechanisms and identifying the key ingredients behind slow relaxation.
\end{abstract}

DOI: 10.1103/PhysRevB.101.174402

\section{INTRODUCTION}

Since the discovery of magnetic hysteresis in $\mathrm{Mn}_{12}$ [1,2], molecular nanomagnets (MNMs) were proposed to reach the ultimate miniaturization of information storage devices. Indeed, their magnetic bistability enables one to encode a bit of information in a single molecule [3,4]. These systems are also ideal test beds for new quantum technologies [5-7], could constitute promising units of future quantum computing architectures [8-14], and were exploited to unveil peculiar quantum phenomena [15-17].

In order to achieve long relaxation times, synthetic efforts are nowadays focusing on transition-metal [18-20] or rare-earth [21] complexes with unquenched orbital angular momentum, which can be characterized by a large anisotropy barrier between the two orientations of the molecular magnetic moment [22]. This led to the synthesis of $4 f$-based single-ion magnets (SIMs) showing slow magnetic relaxation [21,23-31], culminated with the very recent discovery of axial dysprosocenium complexes [32-34] displaying magnetic hysteresis up to $80 \mathrm{~K}$. However, in spite of these great experimental achievements, magnetic relaxation in this new class of compounds [35] is not really understood. A variety of phenomenological formulas have been proposed, but an overarching model able to capture the essential ingredients

\footnotetext{
*david.mills@manchester.ac.uk

†nicholas.chilton@manchester.ac.uk

${ }^{\ddagger}$ stefano.carretta@unipr.it
}

Published by the American Physical Society under the terms of the Creative Commons Attribution 4.0 International license. Further distribution of this work must maintain attribution to the author(s) and the published article's title, journal citation, and DOI. controlling the relaxation dynamics and to explain the observed behavior is still lacking. Such a model is of crucial importance to design new MNMs with even slower relaxation and to exploit these systems for quantum technologies $[6,9,10,12,14]$.

Here we study the $\left[\mathrm{Dy}\left(\mathrm{C}_{5} \mathrm{H}_{2}^{t} \mathrm{Bu}_{3}-1,2,4\right)_{2}\right]\left[\mathrm{B}\left(\mathrm{C}_{6} \mathrm{~F}_{5}\right)_{4}\right]$ dysprosocenium complex (1) [32] as a prototype system to investigate magnetic relaxation in rare-earth SIMs. We disentangle the different relaxation mechanisms, and we derive a model explaining all of them. This is achieved by combining singlecrystal experiments [DC and AC magnetometry, high-field nuclear magnetic resonance (NMR)] with ab initio calculations of the crystal-field (CF) states and of the vibrational modes. In addition, inelastic neutron scattering (INS) is used to validate $a b$ initio calculations of both the static CF and of the phonon density of states (DOS), showing very good agreement. We find that magnetic relaxation in $\mathbf{1}$ arises from the interplay of multistep Orbach, first- and second-order Raman, and quantum tunneling of the magnetization processes. Our model allows us to predict the temperature and field dependence of each mechanism, thus marking the crossover between them, as sketched in Fig. 1. Furthermore, we identify the essential ingredients leading to high blocking temperatures $T_{B}$ (here defined as the temperature where the magnetic relaxation time is equal to $100 \mathrm{~s}[34,36])$ in rare-earth SIMs. In particular, suppressing the efficacy of the Raman mechanism is mandatory in order to set $T_{B}$ in the high- $T$ region, consistent with recent empirical observations [36]. We show that the Raman process is driven by low-energy acoustic phonons and is particularly inefficient in $\mathbf{1}$ thanks to a phonon DOS relatively flat in the range of $\sim 5-20 \mathrm{meV}$, to a large gap $(65 \mathrm{meV})$ between the two lowest crystal-field doublets, and to an ineffective magnetoelastic coupling with optical phonons. These observations provide a route to the synthesis of SIMs with even improved performances. 


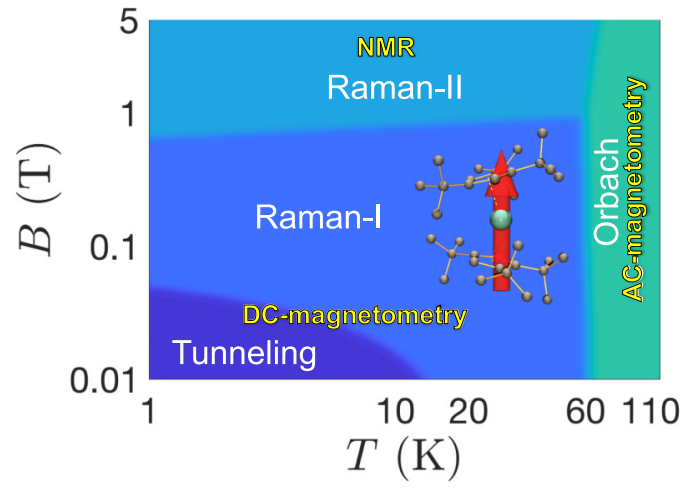

FIG. 1. Sketch of the relaxation mechanisms in $\mathbf{1}$, dominating in different temperature and magnetic field ranges (for $\theta=60^{\circ}$ ). Each process is probed by means of specific experimental techniques, highlighted in yellow. Inset: Molecular structure of the cation of $\mathbf{1}$, with the arrow indicating the easy anisotropy axis.

\section{MODEL}

The Hamiltonian consists of three terms,

$$
H=H_{J}+H_{p}+H_{J p},
$$

representing the magnetic, phonon, and magnetoelastic contributions, respectively. In particular, $H_{J}=V_{\mathrm{CF}}+g_{J} \mu_{B} \mathbf{B} \cdot \mathbf{J}+$ $A \mathbf{J} \cdot \mathbf{I}$, where $V_{\mathrm{CF}}=\sum_{k=2,4,6} \sum_{q=-k}^{k} B_{k}^{q} O_{k}^{q}(\mathbf{J})$ is the crystalfield interaction, expressed as a function of Stevens's operator equivalents $O_{k}^{q}$ acting on the $J=15 / 2$ total angular momentum multiplet of the $\mathrm{Dy}^{3+}$ ion; the $B_{k}^{q}$ coefficients are computed $a b$ initio [32]. The other two terms describe the Zeeman interaction with an external magnetic field $\mathbf{B}$ (with $g_{J}=4 / 3$ ) and the isotropic hyperfine coupling [37], included only for Dy isotopes with spin-active nuclei. The magnetoelastic term is given by

$$
H_{J p}=\zeta_{a 1} H_{J p}^{(a) 1}+\zeta_{a 2} H_{J p}^{(a) 2}+\zeta_{o}\left[H_{J p}^{(o) 1}+H_{J p}^{(o) 2}\right],
$$

accounting for the coupling with acoustic $\left(H_{J p}^{(a) 1,2}\right)$ and optical $\left(H_{J p}^{(o) 1,2}\right)$ phonons up to second order in the strain. We have checked that the specific form of $H_{J p}^{(a)}$ is not crucial for drawing the following general conclusions [38]. Hence, we assume for it the widely used "rotational Debye model" [39-41] (see Appendix A), with first- and second-order terms proportional to $\sum_{\alpha} \sum_{\mathbf{k} \lambda} \sqrt{|\mathbf{k}|}\left[V_{\mathrm{CF}}, J_{\alpha}\right]$ and $\sum_{\alpha \beta} \sum_{\mathbf{k} \lambda \mathbf{k}^{\prime} \lambda^{\prime}} \sqrt{|\mathbf{k}||\mathbf{k}|^{\prime}}\left[\left[V_{\mathrm{CF}}, J_{\alpha}\right], J_{\beta}\right]$, respectively [42], with $(\mathbf{k}, \lambda)$ being the wave vector and branch index of each phonon mode. Conversely, optical phonons are described as nondispersive local modes [43,44] (see Appendix A) with $H_{J p}^{(o) 1}=\sum_{k, q} \sum_{\Gamma} \frac{1}{\sqrt{\omega_{\Gamma}}} \frac{\partial B_{k}^{q}}{\partial u_{\Gamma}}\left(a_{\Gamma}^{\dagger}+a_{\Gamma}\right) O_{k}^{q}$, where $u_{\Gamma}$ is the atomic displacement along the normal mode $\Gamma$ with energy $\hbar \omega_{\Gamma}$ and $a_{\Gamma}^{\dagger}$ and $a_{\Gamma}$ are the corresponding creator and annihilator operators, respectively. Here the magnetoelastic couplings containing the derivatives $\frac{\partial B_{k}^{q}}{\partial u_{\Gamma}}$ are computed ab initio [32] for each term of the expansion into Stevens's equivalent operators $O_{k}^{q}$, acting on the electronic system. The calculated $H_{J p}^{(o) 2}$ [32] gives a very small contribution to the observed relaxation and is neglected hereafter. Thus, the $\zeta$ factors in (2) are the only fitting parameters of our model.

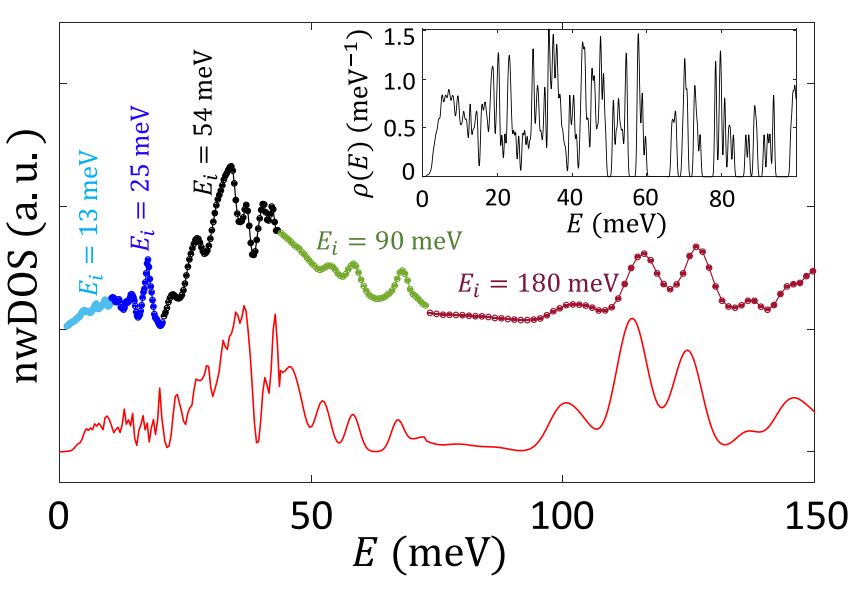

FIG. 2. Comparison between measured (symbols, $T=5 \mathrm{~K}$ ) and calculated (red line) neutron-weighted phonon density of states. Different colors in the experimental nwDOS refer to different incident energies $E_{i}$ and resolutions. Inset: Calculated phonon DOS $\rho(E)$ with single FWHM $=0.29 \mathrm{meV} . \rho(E)$ is normalized to the total number of atoms $N$ in the unit cell, $\int \rho(E) d E=3 N$.

The predicted magnetic spectrum is tested by INS measurements performed on the MERLIN spectrometer at ISIS [45], exploring energies up to $155 \mathrm{meV}$. A single magnetic excitation is found at $\approx 65 \mathrm{meV}$ (see Fig. S3 [38]), in very good agreement with the $a b$ initio prediction of the first excited CF doublet (58 meV) [32]. The absence of other magnetic peaks also supports the axial character of the predicted $\mathrm{CF}$, yielding a ground doublet with $g_{\perp} \approx 0$ and $g_{z} \approx 20$. Hereafter, we indicate with $z$ the easy axis of $V_{\mathrm{CF}}$ (see the molecular structure in the inset of Fig. 1). In addition, we show in Fig. 2 the neutron-weighted phonon DOS (nwDOS) obtained from INS measurements. In the one-phonon incoherent approximation the INS cross section can be expressed as $S_{\text {inc }}(Q, E) \propto \frac{Q^{2}}{E}\left[\sum_{l} \frac{\sigma_{l}^{\text {scatt }}}{2 m_{l}} \rho_{l}(E)\right]$, where the term in square brackets is the nwDOS, $\rho_{l}(E)$ is the partial DOS for element $l, \sigma_{l}^{\text {scatt }}$ is the scattering cross section, and $m_{l}$ is the atomic mass. Hence, although the phonon DOS $\rho(E)=\sum_{l} \rho_{l}(E)$, needed to compute the system relaxation dynamics, is not directly accessible by INS, we can use the partial DOS $\rho_{l}$ determined $a b$ initio to reconstruct the nwDOS, compare with experiment, and check the reliability of $a b$ initio calculations. Results reported in Fig. 2 show excellent agreement between the nwDOS computed by periodic density functional theory (DFT) calculations $[46,47]$ and that measured by INS in the whole energy range (which has been reconstructed by merging the results of several INS data sets with different experimental configurations [38]). This demonstrates the reliability of $a b$ initio calculations and allows us to use the phonon DOS $\rho(E)$ from theory (reported in the inset of Fig. 2) to compute the relaxation dynamics.

\section{DISENTANGLING RELAXATION MECHANISMS}

Starting from the eigenstates of $H_{J}$, we compute magnetic relaxation rates by considering both resonant and nonresonant processes, using the phonon DOS computed $a b$ initio and validated by INS. As detailed below and sketched in Fig. 1, 
we find that resonant Orbach processes dominate at high temperature, where optical phonons resonant with CF gaps are available. For $T \lesssim 60 \mathrm{~K}$ first- and second-order Raman rates emerge in different field ranges, while quantum tunneling dominates in the low- $T$, low- $B$ region. These mechanisms are disentangled by specific single-crystal experiments, as indicated in Fig. 1.

We start from the intermediate-temperature range $(15 \lesssim$ $T \lesssim 60 \mathrm{~K}$ ) dominated by the Raman mechanism (see Fig. 1) because this process is crucial to achieve a high $T_{B}$ (see below). In this region, the system dynamics is restricted to the lowest Kramers doublet, and excited CF states contribute only via nonresonant first- and second-order Raman processes. The corresponding rate is proportional to the integral (see the Supplemental Material (SM) [38] and Refs. [39,48,49]):

$$
\int_{0}^{\infty} \frac{d \omega e^{\hbar \omega / k_{B} T}}{\left(e^{\hbar \omega / k_{B} T}-1\right)^{2}} \rho(\hbar \omega) \rho(\hbar \omega+\Delta)\left|M_{I}+M_{I I}\right|^{2},
$$

where $M_{I}\left(M_{I I}\right)$ contains matrix elements of $H_{J p}^{1}\left(H_{J p}^{2}\right)$, associated with the Raman-I (Raman-II) process [39]. In particular, $M_{I}$ arises from treating $H_{J p}^{1}$ to second order in time-dependent perturbation theory and thus contains contributions inversely proportional to the difference between crystal field gaps and phonon energies. Conversely, the Raman-II mechanism is obtained by applying first-order time-dependent perturbation theory to the second-order magnetoelastic coupling $H_{J p}^{2}$ (see Appendix A and the SM [38]). Calculating integral (3), we find that in the intermediate-temperature range the relaxation rate is

$$
\tau_{\mathrm{IT}}^{-1}=\frac{B_{z}^{2}+\frac{\mu}{2} \sigma_{B}^{2}}{B_{z}^{2}+\frac{1}{2} \sigma_{B}^{2}}\left(c_{1} T^{n}+c_{2} B^{2} T^{m}\right),
$$

where the two terms in parentheses account for Raman-I and Raman-II mechanisms and the coefficients $c_{1,2}$ depend on $\zeta$ parameters in (2). $n$ and $m$ are mostly determined by the phonon DOS and the lowest CF gap, and we find the small values $n=2.3$ and $m=2.1$, which reproduce very well the experimental temperature dependence (see below). As far as the magnetic field dependence is concerned, we find a Raman-II contribution to $\tau_{\mathrm{IT}}^{-1}$ approximately proportional to $B^{2}$ and a Raman-I contribution independent of $B$ [38], as expected for a Kramers system [39]. The additional BronsVan Vleck prefactor $[48,50] \frac{B_{z}^{2}+\mu \sigma_{B}^{2} / 2}{B_{2}^{2}+\sigma_{B}^{2} / 2}$ [38] accounts for the effect of the internal field due to the surrounding nuclear and electronic spins [51]. Since $\mu$ and $\sigma_{B}$ depend on the unknown distribution of such internal fields, we have kept them as fitting parameters. A significant dependence of the relaxation rate on the presence of Dy magnetic nuclei for $B_{z}>20 \mathrm{mT}$ has been ruled out by calculations involving hyperfine interactions [38], in agreement with a recent study on an isotopically enriched Dy complex [35].

Out-of-equilibrium magnetization measurements have been performed by preparing the sample in zero field and then measuring the magnetization $M$ as a function of time after switching on $\mathbf{B}$ in the range $0.005<B<2 \mathrm{~T}$. Most of the measurements have been performed at a fixed angle $\theta \approx 60^{\circ}$ between the field and the molecular easy axis to investigate relaxation in the presence of sizable $B_{x}$ and $B_{z}$. We have finally tested our results with some measurements at $\theta \approx 0^{\circ}$.
The observed time dependence of $M$ has been fitted by a stretched exponential function, which points to a small strain in the CF parameters, yielding an asymmetric distribution of relaxation times (see Appendix B and Ref. [52]). The extracted relaxation rates are shown in Fig. 3(a) as a function of the applied field. These and all the experimental findings reported in the following and in the SM are very well reproduced by using $\zeta_{a 1}=1.8 \times 10^{-9} \mathrm{~s}^{1 / 2}, \zeta_{a 2}=1.1 \times 10^{-16} \mathrm{~s}$, and $\zeta_{o}=0.9 \AA \mathrm{s}^{-1 / 2}$ in Eq. (2) (see solid lines in Fig. 3). The decrease of $\tau^{-1}$ at $\sim 0.1 \mathrm{~T}$ is due to intermolecular dipole fields modeled by the Brons-Van Vleck term $\left(\sigma_{B}=30 \mathrm{mT}\right.$, $\mu=3$ ) [48], while the increase above $0.3 \mathrm{~T}$ is due to the Raman-II mechanism, with $\tau^{-1} \propto B^{2}$. Measurements at $\theta \approx$ $0^{\circ}$ are reported in green and evidence a modest slowdown of the dynamics, consistent with a decrease of the transverse component of $\mathbf{B}$ [54]. The increase of $\tau^{-1}$ above $\sim 0.3 \mathrm{~T}$ in Fig. 3(a) suggests that Raman-II becomes the leading mechanism at high fields, a regime which can be investigated by NMR measurements. Therefore, we have measured the proton spin-lattice relaxation rate $1 / T_{1}$ as a function of temperature. Part of the data is reported in Fig. 3(b) for two different values of $B$ at $\theta \approx 60^{\circ}$. It can be shown [55] (Appendix D) that in the present regime (characterized by an electronic relaxation rate much smaller than the proton Larmor frequency) $1 / T_{1} / \chi T$ is $\propto \tau^{-1} / B^{2}$. Figure 3(b) shows that data collected for different $B$ are practically superimposed. Thus, in the temperature and field region probed by NMR $\tau^{-1} \propto B^{2}$, demonstrating that Raman-II is the leading relaxation mechanism (see Fig. 1) [56], consistent with our prediction. This conclusion is further supported by the power-law temperature dependence of $1 / T_{1}$ below $60 \mathrm{~K}$, in excellent agreement with our model, yielding $m=2.1$ [solid lines in Fig. 3(b)]. Another data set collected at $\theta \approx 90^{\circ}$ and $B=2.5-5 \mathrm{~T}$ is reported in the SM [38], showing analogous agreement with our model.

At lower fields, our model and data in Fig. 3(a) indicate that the Raman-II contribution is overcome by the RamanI (field-independent) mechanism. This is confirmed by the temperature dependence of $\tau^{-1}$ extracted from magnetization measurements at $B=0.1 \mathrm{~T}$ [Fig. 3(c)], showing a power-law behavior for $T \leqslant 50 \mathrm{~K}$, very well reproduced by our simulation (with $n=2.3$ ). These results are also in good agreement with powder data on the same compound [32]. The set of relaxation coefficients calculated from the model [Eq. (2)] is listed in the table in Fig. 3(g). The measured temperature and field dependence of $\tau^{-1}$ (see Fig. 3 and Appendix C) exclude a significant direct contribution (arising, e.g., from a modulation of the Zeeman or hyperfine interaction [57]), which would require the presence of phonons of energy corresponding to the splitting of the ground doublet $\Delta=g_{z} \mu_{B} B_{z}$, yielding $\tau^{-1} \propto B^{5} \operatorname{coth}\left(\Delta / 2 k_{B} T\right) \approx B^{4} T$ [37]. This is particularly evident by examining data collected in the high-field region: the field dependence extracted from magnetization data, as well as the temperature dependence obtained from NMR at different values of $B$, cannot be reproduced by assuming a direct instead of a Raman-II contribution (see Figs. 6 and 7).

At higher temperatures, simulating the relaxation dynamics requires consideration of the rate matrix $\mathbb{W}$ involving all 16 levels of the $J=15 / 2$ multiplet (see Appendix D), including real transitions to excited CF states. By diagonalizing $\mathbb{W}$ we find that a single relaxation rate dominates the dynamics, 

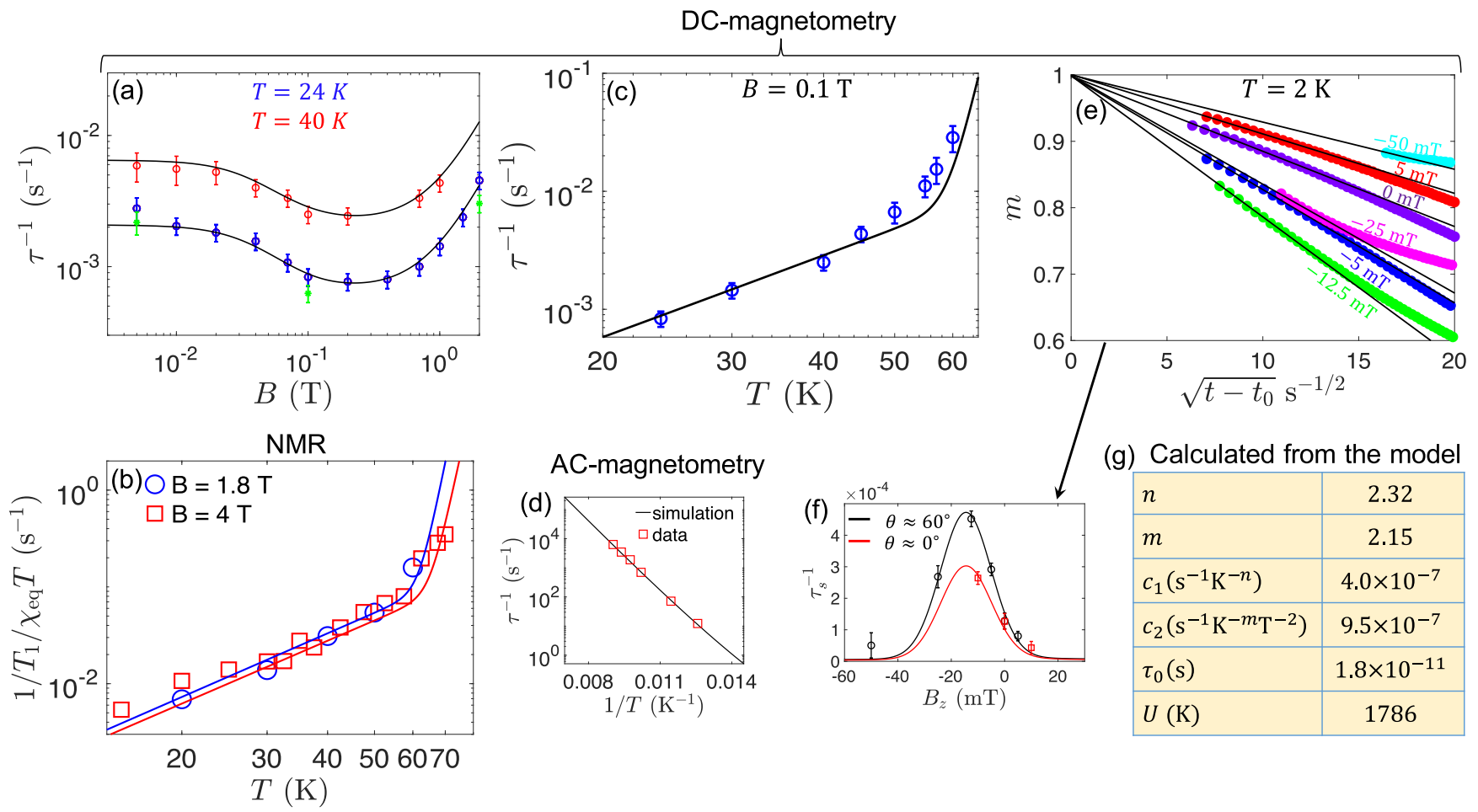

(g) Calculated from the model

\begin{tabular}{|l|c|}
\hline$n$ & 2.32 \\
\hline$m$ & 2.15 \\
\hline$c_{1}\left(\mathrm{~s}^{-1} \mathrm{~K}^{-n}\right)$ & $4.0 \times 10^{-7}$ \\
\hline$c_{2}\left(\mathrm{~s}^{-1} \mathrm{~K}^{-m} \mathrm{~T}^{-2}\right)$ & $9.5 \times 10^{-7}$ \\
\hline$\tau_{0}(\mathrm{~s})$ & $1.8 \times 10^{-11}$ \\
\hline$U(\mathrm{~K})$ & 1786 \\
\hline
\end{tabular}

FIG. 3. (a) Magnetic-field dependence of $\tau^{-1}$ extracted from DC magnetization measurements as a function of the external field at 24 (blue symbols) and $40 \mathrm{~K}$ (red) and $\theta \approx 60^{\circ}$. Lines are the corresponding calculation. Green circles represent relaxation rates collected at $24 \mathrm{~K}$ and $\theta \approx 0^{\circ}$. (b) NMR proton relaxation rates $\left(1 / T_{1}\right)$ as a function of $T$ at different fields for $\theta \approx 60^{\circ}$. Errors are within the points' dimension. Solid lines are the corresponding simulations, with Raman mechanisms dominating below 55-60 K and Orbach process becoming effective at high $T$. (c) Temperature dependence of $\tau^{-1}$ extracted from DC magnetization measurements for $B=0.1 \mathrm{~T}$ and $\theta \approx 60^{\circ}$ (points) and simulated (line). (d) Relaxation rates extracted from AC magnetization measurements (squares) and calculated from diagonalization of the rate matrix (line) in zero field. (e) Magnetization decay at low temperature and low field for $\theta \approx 60^{\circ}$. Solid lines are fit at short times according to $m(t)=1-\sqrt{\left(t-t_{0}\right) / \tau_{s}}$, from which the short-time relaxation rate $\tau_{s}$ is extracted. Magnetization is normalized to the saturation value, $m=M / M_{\text {sat }}$. (f) Dependence of $\tau_{s}$ on the component of the external field along the easy axis, proportional to the initial normalized distribution of energy bias in the sample, here modeled by a Gaussian with $\sigma_{B}=9.5 \mathrm{mT}$ and $B_{z}^{l}=14.5 \mathrm{mT}$ [53]. (g) Relaxation coefficients, calculated from the model [Eq. (2)], with $\zeta$ parameters fitted from the data.

yielding an Arrhenius-like behavior: $\tau_{\mathrm{HT}}^{-1}=\tau_{0}^{-1} e^{-U / k_{B} T}$, in excellent agreement with AC-susceptibility measurements reported in Fig. 3(d). This process is driven by optical phonons and enables us to determine $\zeta_{o}$ in Eq. (2). The calculated $\tau_{\mathrm{HT}}^{-1}$ is then characterized by $\tau_{0}=1.8 \times 10^{-11} \mathrm{~s}$ and $U=1786 \mathrm{~K}$ [Figs. 3(d) and 3(g)], in excellent agreement with powder data [32].

Finally, the onset of a temperature-independent tunneling regime is evidenced below $15 \mathrm{~K}$ both by powder and singlecrystal measurements reported in Fig. 3(e) and in the SM [38], with relaxation rates $\sim 3-4$ orders of magnitude smaller than for another recently studied Dy ${ }^{3+}$ complex [35]. At short times, we find the $\sqrt{t}$ magnetization decay predicted in Ref. [58], and we extract the peculiar $\mathbf{B}$ dependence of the relaxation rate. This is reported in Fig. 3(f) as a function of $B_{z}$ for two different data sets corresponding to different $\theta$ (see also Fig. S19 [38]). The short-time relaxation rate $\tau_{s}{ }^{-1}$ is proportional to the tunnel splitting [59] and to the initial distribution $P\left(B_{z}\right)$ of internal fields, modeled by a Gaussian function [60]: $\tau_{s}^{-1}=c \frac{\Omega^{2}}{\hbar} P\left(B_{z}\right)=c \frac{\Omega^{2}}{\hbar \sqrt{2 \pi} \sigma_{B}} e^{-\frac{\left(B_{z}+B_{2}\right)_{2}^{2}}{2 \sigma_{B}}}$, where $\Omega$ is the tunnel splitting, proportional to the total (internal + external) transverse field [59], and $B_{z}^{l}$ accounts for internal hyperfine and dipole fields [61]. Fitting these rates, we find $B_{z}^{l}=14.5 \mathrm{mT}$, in agreement with that reported for a similar Dy complex [35] and with the estimated dipolar field due to neighboring molecules. Remarkably, data collected at $\theta \approx$ $60^{\circ}$ and $0^{\circ}$ show the same behavior as a function of $B_{z}$ [Fig. 3(f)], with a lower amplitude due to the smaller tunnel splitting (because $\mathbf{B}$ is almost parallel to $z$ ). Note that even for $\theta \approx 0^{\circ}$ transverse internal fields (which we estimate to be of the order of tens of milliteslas, consistent with other MNMs [62]) still induce tunneling.

\section{DISCUSSION}

We now discuss the key ingredients explaining the behavior of $\mathbf{1}$ in order to identify strategies to realize highblocking-temperature molecular storage devices. The crucial requirement is to reduce the efficiency of Raman relaxation, thus increasing $T_{B}$ up to the Orbach region. The integral (3) involves both the acoustic $\left(0<\omega<\omega_{D}\right)$ and optical $(\omega>$ $\left.\omega_{D}\right)$ parts of the phonon spectrum, with $\hbar \omega_{D}=4.4 \mathrm{meV}$, determined from the phonon DOS [38]. First, we note that due to the form of integrand (3) (strongly decreasing with energy [63]) Raman relaxation is driven by acoustic phonons. 


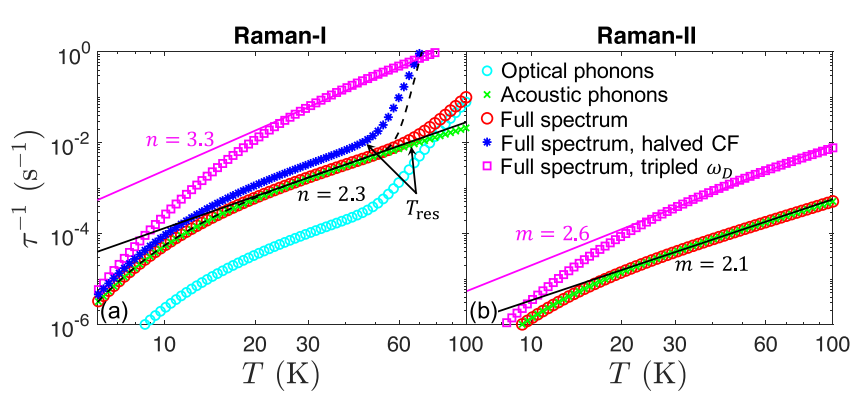

FIG. 4. Contributions to (a) Raman-I and (b) Raman-II relaxation rates at $0.1 \mathrm{~T}, \theta=60^{\circ}$. Colored symbols are calculated with our model, while solid lines are fits in the high-temperature regions using a power law, whose exponent is reported on the figure. The dashed line in (a) also includes a multistep Orbach contribution.

This is evident by comparing the calculations reported with green and red symbols in Fig. 4; the former are obtained by considering only the contribution of acoustic phonons, and the latter by including the whole phonon spectrum. For $T \lesssim 70 \mathrm{~K}$, the two curves are practically superimposed and show a power-law behavior (black solid line) with $n=2.3$ and $m=2.1$, while the contribution of optical phonons [light blue symbols in Fig. 4(a)] is negligible. At a higher temperature [ $T_{\text {res }}$ in Fig. 4(a)], a resonant two-step process emerges in the Raman-I mechanism. This results from optical phonons at energies close to the lowest crystal-field gap $\Delta_{\mathrm{CF}} \approx 60 \mathrm{meV}$, leading to a divergence in the denominator of $M_{I}$ from secondorder perturbation theory [38] and hence an Orbach-like temperature dependence $\tau^{-1} \sim e^{-\Delta_{\mathrm{CF}} / k_{B} T}$. Here $T_{\text {res }} \approx 70 \mathrm{~K}$ [red symbols in Fig. 4(a)]; that is, the transition to this regime would occur in a region where Raman-I is already overcome by the multistep Orbach process (dashed line and Fig. 1).

Suppression of Raman relaxation (as observed in 1) can be obtained by combining several ingredients [all reducing integral (3)]: (i) a large crystal-field splitting between the two lowest Kramers doublets, which can be engineered by a judicious choice of molecular geometry, and (ii) a relatively small magnetoelastic coupling with low-energy optical modes, meaning that vibrations mostly influencing the local $\mathrm{CF}$ at Dy should be high in energy (above $\sim 30 \mathrm{meV}$ in 1; see Fig. S1 [38]). This occurs in the presence of a rigid first-coordination sphere. Features (i) and (ii) increase $T_{\text {res }}$, thus making optical phonons ineffective for Raman-I. For instance, $T_{\text {res }}$ is reduced both by reducing $\Delta_{\mathrm{CF}}$ [compare the dark blue curve in Fig. 4(a), obtained by halving the CF parameters, with the original (red) one] and by increasing $\zeta_{o}$. The last ingredient to suppress Raman relaxation is (iii) a rather small $\hbar \omega_{D}$ followed by a plateau in the DOS (i.e., an optical phonon DOS not strongly increasing with energy at low energies), which arises here due to high-energy intramolecular modes and weak intermolecular interactions. Indeed, for $T>20 \mathrm{~K}$ both Raman-I and Raman-II contributions show a weak power-law temperature dependence, with $n=2.3 \approx m=2.1$, close to the $T^{2}$ behavior corresponding to the high-temperature limit of Raman mechanisms [43,50]. An increase in $\omega_{D}$ shifts the crossover between low- and high- $T$ limits to higher temperatures, as shown by the magenta curves in Fig. 4, increasing $n(m)$ to 3.4 (2.6). A similar effect is obtained with an optical $\rho(E)$ strongly increasing with energy at low energy or with highly dispersive low-energy optical modes.

Once Raman relaxation is suppressed, further improvements rely on increasing $\tau_{0}$ and $U$ in the Orbach process, driven by optical phonons. This can be achieved by engineering molecular vibrational modes [to increase $\tau_{0}$ by reducing the magnetoelastic coupling or $\rho(E)$ at the energies corresponding to $\mathrm{CF}$ gaps] or by chemically designing molecules with larger CF splittings and more axial Kramers doublets (thus increasing $U$ ). $\mathrm{CF}$ engineering to increase $U$ is much more efficient than tailoring phonon modes, as demonstrated by the recent record hysteresis temperature of $80 \mathrm{~K}$ [33].

In conclusion, we have developed a model for magnetic relaxation in high-blocking-temperature SIMs based on targeted single-crystal magnetization and NMR measurements, neutron spectroscopy, and ab initio calculations. We have disentangled the various mechanisms behind the complex relaxation phenomenon in rare-earth SIMs and highlighted the peculiar temperature and field dependence of each mechanism, catching the essential ingredients leading to slow magnetic relaxation in these compounds. We have used this information to provide rules for reducing the effectiveness of the Raman mechanism and thus increasing the blocking temperature. The general recipe is a crystal of weakly interacting rigid molecules in order to have soft acoustic branches and high-energy optical modes.

\section{ACKNOWLEDGMENTS}

Experiments at the ISIS Neutron and Muon Source were supported by beam time allocation RB1810337 (https://doi. org/10.5286/ISIS.E.92923034) from the Science and Technology Facilities Council. This work was partly funded by the Italian Ministry of Education and Research (MIUR) through PRIN Project 2015 HYFSRT "Quantum Coherence in Nanostructures of Molecular Spin Qubits" and by the European Project "Scaling up quantum computation with molecular spins" (SUMO) of the call QuantERA, by the U.K. EPSRC (Doctoral Prize Fellowship to C.A.P.G., EP/P002560/1 to D.R. and F.O.), and by the University of Manchester. We thank the EPSRC National Research Facility for electron paramagnetic resonance spectroscopy for access to the superconducting quantum interference device magnetometer. M.G. thanks The University of Manchester for a Presidential Doctoral Scholarship and the MOLSPIN COST action CA15128. N.F.C. thanks The University of Manchester for a Presidential Fellowship and the Royal Society for a University Research Fellowship. E.M. and E.G. acknowledge the support of the PRISM Project of the call "FIL-Quota incentivante 2019" of the University of Parma.

\section{APPENDIX A: MAGNETOELASTIC INTERACTION}

Our theoretical model is based on a minimal form of the magnetoelastic interaction in which acoustic phonons are modeled in the Debye approximation, while optical modes are considered local and hence their phonon dispersion is neglected, as assumed in Refs. [39-41,43,64-66]. Our aim here is not to obtain deep insight into molecular vibrations, 
but rather to highlight the essential ingredients behind slow magnetic relaxation, which already emerge in our minimal treatment based on Refs. [39-41,43,64-66]. We adopt the harmonic approximation for lattice vibrations, i.e., $H_{p}=$ $\sum_{\mathbf{k} \lambda} \hbar \omega_{\mathbf{k} \lambda}\left(a_{\mathbf{k} \lambda}^{\dagger} a_{\mathbf{k} \lambda}+\frac{1}{2}\right)$, where $a_{\mathbf{k} \lambda}^{\dagger}\left(a_{\mathbf{k} \lambda}\right)$ creates (destroys) a phonon of wave vector $\mathbf{k}$ in the $\lambda$ th phonon branch with energy $\hbar \omega_{\mathbf{k} \lambda}$ [42]. For simplicity, we assume that the magnetoelastic interaction involving acoustic (dispersive) phonons is driven by the "rotational contribution," which changes the direction of anisotropy axes while keeping the molecule rigid $[39,41]$. We also assume an isotropic phonon space $\left(k_{x}=k_{y}=k_{z}\right)$ [43] with Debye dispersion $\omega_{\mathbf{k} \lambda}=v|\mathbf{k}|$ and sound velocity $v \equiv v_{x}=v_{y}=v_{z}$. We stress, however, that our conclusions are not affected by this assumption. In this approximation, we get [42]

$$
\begin{aligned}
H_{J p}^{a(1)}= & \sum_{\alpha=x, y, z} \sum_{\mathbf{k} \lambda} \sum_{k, q} \xi_{\mathbf{k} \lambda}^{\alpha} B_{k}^{q}\left[O_{k}^{q}, J_{\alpha}\right]\left(a_{\mathbf{k} \lambda}^{\dagger}+a_{-\mathbf{k} \lambda}\right), \\
H_{J p}^{a(2)}= & \sum_{\substack{\alpha, \beta=\\
x, y, z}} \sum_{\mathbf{k} \lambda} \sum_{k, q} \xi_{\mathbf{k} \lambda \mathbf{k}^{\prime} \lambda^{\prime}}^{\alpha \beta} B_{k}^{q}\left[\left[O_{k}^{q}, J_{\alpha}\right], J_{\beta}\right] \\
& \times\left(a_{\mathbf{k} \lambda}^{\dagger}+a_{-\mathbf{k} \lambda}\right)\left(a_{\mathbf{k}^{\prime} \lambda^{\prime}}^{\dagger}+a_{-\mathbf{k}^{\prime} \lambda^{\prime}}\right),
\end{aligned}
$$

where

$$
\begin{aligned}
\xi_{\mathbf{k} \lambda}^{\alpha} & =i e^{i \mathbf{k} \cdot \mathbf{r}}\left(\hat{\mathbf{k}} \times \hat{\mathbf{e}}_{\mathbf{k} \lambda}\right)_{\alpha} \sqrt{\omega_{\mathbf{k} \lambda}}, \\
\xi_{\mathbf{k} \lambda \mathbf{k}^{\prime} \lambda^{\prime}}^{\alpha \beta} & =-e^{i\left(\mathbf{k}+\mathbf{k}^{\prime}\right) \cdot \mathbf{r}}\left(\hat{\mathbf{k}} \times \hat{\mathbf{e}}_{\mathbf{k} \lambda}\right)_{\alpha}\left(\hat{\mathbf{k}}^{\prime} \times \hat{\mathbf{e}}_{\mathbf{k}^{\prime} \lambda^{\prime}}\right)_{\beta} \sqrt{\omega_{\mathbf{k} \lambda} \omega_{\mathbf{k}^{\prime} \lambda^{\prime}}},
\end{aligned}
$$

with $\hat{\mathbf{k}}=\mathbf{k} /|\mathbf{k}|$ and $\hat{\mathbf{e}}_{\mathbf{k} \lambda}$ being the polarization unit vector of mode $\{\mathbf{k} \lambda\}$. Since this is a simplified form of the magnetoelastic coupling which does not really take into account the internal structure of the molecule (modeled as rigid), we have included two different scale factors, $\zeta_{a 1}$ and $\zeta_{a 2}$ in Eq. (2), between first- and second-order contributions. These are kept as fitting parameters to account for the limits of such an approximation.

In the case of local phonon modes [43] or optical phonons with negligible dispersion $[43,67]$, the first-order magnetoelastic Hamiltonians reads

$$
H_{J p}^{o(1)}=\sum_{k, q} \sum_{\Gamma} \eta_{\Gamma}^{k q}\left(a_{\Gamma}^{\dagger}+a_{\Gamma}\right) O_{k}^{q}, \quad \eta_{\Gamma}^{k q}=\frac{1}{\sqrt{\omega_{\Gamma}}} \frac{\partial B_{k}^{q}}{\partial u_{\Gamma}} .
$$

Here $u_{\Gamma}$ are atomic displacements along the local normal mode $\Gamma$, and the coefficients $\eta_{\Gamma}^{k q}$ are obtained from ab initio calculations [32] by considering the derivatives of $B_{k}^{q}$ induced by atomic displacements of the ligands along the normalmode $\Gamma$ computed in the gas phase, as well as their energies (rescaled by comparison to Raman and infrared spectra). It is worth noting that the inclusion of a small dispersion [43] for optical modes does not change the conclusions of this work.

\section{APPENDIX B: ORIGIN OF THE STRETCHED EXPONENTIAL BEHAVIOR}

Magnetization data (reported in Figs. S8-S12 [38]) show stretched-exponential behavior $M(t)=M(0)+\left[M_{\text {sat }}-\right.$ $M(0)]\left[1-e^{-(t / \tau)^{\beta}}\right]$, with $M_{\text {sat }} \propto g_{z} B_{z} \tanh \frac{g_{z} \mu_{B} B_{z}}{2 k_{B} T}$ and $\beta \sim$ $0.8-0.85$. Such stretched-exponential behavior points to an asymmetric distribution of relaxation times [52]. This can
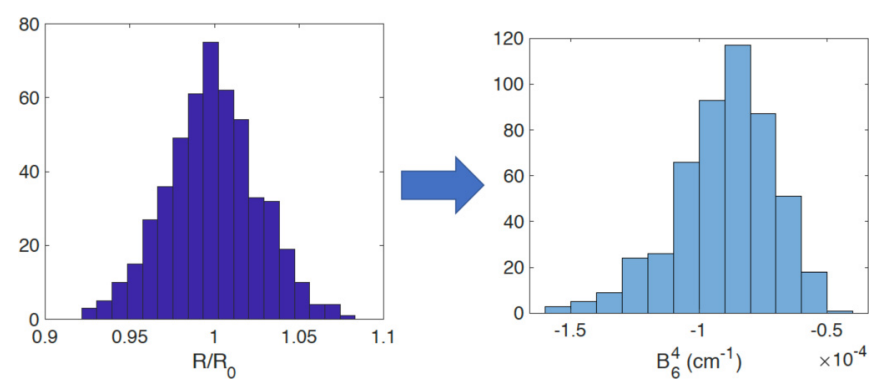

FIG. 5. Left: Gaussian distribution of relative distortions of the ligands from their equilibrium position with a standard deviation of 0.03. Right: Resulting (asymmetric) distribution of the CF parameter in the point charge approximation.

be understood by considering that different molecules in the crystal will be characterized by slightly different distances between the magnetic ion and the surrounding ligands. This, in turn, leads to slightly different CF parameters for each molecule and hence to a corresponding distribution of relaxation times.

To qualitatively understand the effect of such a distribution, we have drawn a minimal model in which all the ligands are uniformly distorted with a relative strain $\delta R / R_{0}$ with respect to their average position $R_{0}$. We have numerically simulated the effect of such a distribution of distances on the CF parameters $B_{k}^{q}$ by assuming a Gaussian distribution of $\delta R / R_{0}$ and a point charge model for $B_{k}^{q}$ [37]. Within this approximation

$$
B_{k}^{q} \propto \sum_{i} \frac{Z_{i}}{R_{i}^{k+1}} \sim \sum_{i} \frac{Z_{i} R_{0 i}^{-k-1}}{\left(1+\frac{\delta R_{i}}{R_{0 i}}\right)^{k+1}} \sim \frac{\left(B_{k}^{q}\right)_{0}}{\left(1+\frac{\delta R}{R_{0}}\right)^{k+1}},
$$

and hence, we expect an asymmetric distribution of CF parameters even starting from a symmetric distribution of $\delta R / R_{0}$. In particular, we have numerically checked that a distribution of $\delta R / R_{0}$ with a standard deviation of 3\%-5\% (a reasonable assumption for this class of compounds) is sufficient to produce a stretched exponential decay with $\beta \sim 0.8$ by varying only a single coefficient at a time (see Fig. 5). Indeed, by computing the spread of relaxation rates corresponding to the distribution of $B_{k}^{q}$, we have found it to be also asymmetric and reproducible with a stretched-exponential function with $\beta \sim$ 0.8 . We have checked that qualitatively similar distributions of relaxation rates are obtained for different forms of the magnetoelastic coupling, temperature, magnetic fields, and initial distributions of the CF parameters and of the initial magnetization. Hence, this simple argument gives a qualitative explanation of the observed form of the magnetization decay in $\mathbf{1}$, as in a large class of similar compounds [33,35].

\section{APPENDIX C: DIRECT RELAXATION MECHANISM}

We report below a comparison between experimental data and the calculated relaxation rate in which the Raman-II mechanism has been replaced by the direct process, yielding $\tau^{-1} \propto B^{5} \operatorname{Coth}_{\frac{z}{z}} \mu_{B} B_{z} k_{B}$ (no approximation has been done on the Bose-Einstein factor). Experimental data in the high-field region (both magnetization, reported in Fig. 6, and NMR, reported in Fig. 7) clearly exclude the occurrence of a sizable direct process. 


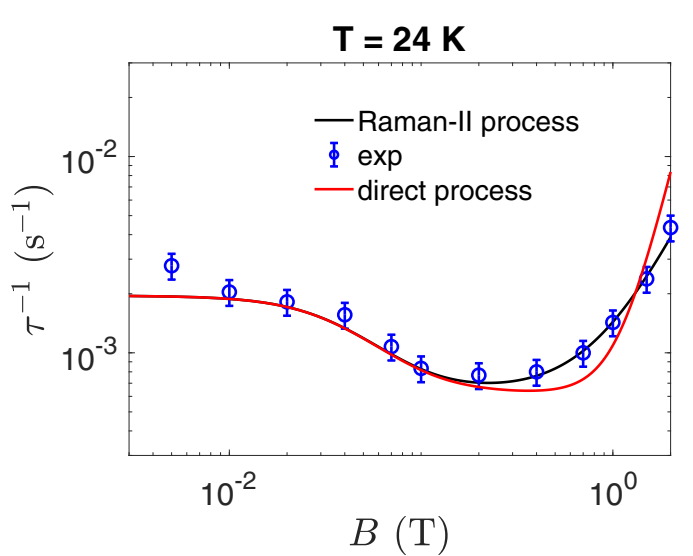

FIG. 6. Field dependence of the relaxation rate, fitted either by assuming a Raman-II mechanism (black curve) or a direct process (red curve).

\section{APPENDIX D: AC SUSCEPTIBILITY}

The high-temperature region of Fig. 1 has been investigated by AC-susceptibility measurements (see Fig. 8). In these experiments, we probe the quasielastic dynamic susceptibility of the system, subject to a small longitudinal field perturbing the equilibrium condition. This can be modeled as $[55,68]$

$$
\begin{aligned}
\chi(\omega)= & \chi_{\mathrm{eq}}(\omega)-i \omega \sum_{s, t}\left(\left\langle s\left|J_{z}\right| s\right\rangle-\left\langle J_{z}\right\rangle_{\mathrm{eq}}\right) \\
& \times \frac{1}{i \omega-\mathbb{W}}\left(\left\langle t\left|J_{z}\right| t\right\rangle-\left\langle J_{z}\right\rangle_{\mathrm{eq}}\right) \frac{p_{t}}{k_{B} T},
\end{aligned}
$$

in which (due to the axiality of the system) we consider only the longitudinal component $\left(\chi \equiv \chi_{z z}\right)$. Here $E_{t}(t=$ $1, \ldots, 16)$ are eigenvalues of the single-ion Hamiltonian, $p_{t}=e^{-E_{t} / k_{B} T} / Z$ is the population of eigenstate $|t\rangle$ at thermal equilibrium, and $\left\langle J_{z}\right\rangle_{\mathrm{eq}}=\sum_{m}\left\langle s\left|J_{z}\right| s\right\rangle p_{m}$. W is the system rate matrix:

$$
W_{s \leftarrow t}=\frac{2 \pi}{\hbar} \zeta^{2} \rho\left(\Delta_{s t}\right) n\left(\Delta_{s t}\right)\left|\left\langle s\left|V_{1}^{\Gamma}\right| t\right\rangle\right|^{2},
$$

where $\Delta_{s t}=E_{s}-E_{t}, \rho\left(\Delta_{s t}\right)$ is the phonon DOS at the frequency corresponding to the gap $\Delta_{s t}, n(x)=\left[e^{x / k_{B} T}-\right.$ $1]^{-1}$ is the Bose-Einstein factor, and we consider in $V_{1}^{\Gamma}=$ $\sum_{k, q} \eta_{\Gamma}^{k q} O_{k}^{q}$ the modulation induced by the calculated phonon mode $\Gamma$ closest in energy to $\Delta_{s t}$ (using the "calibrated" mode energies from the DFT [32]). Notice that only optical phonon
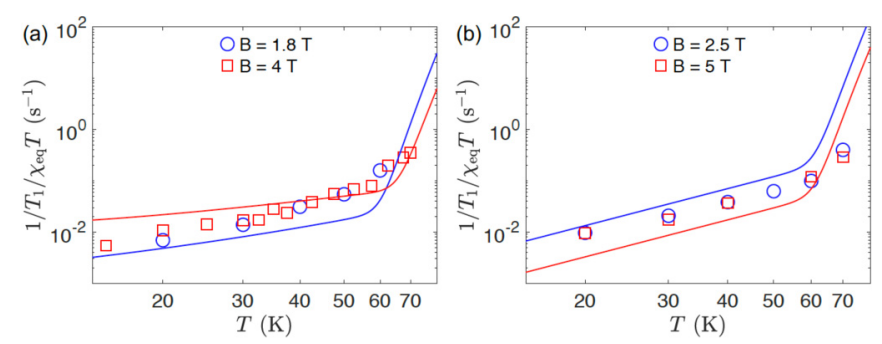

FIG. 7. Temperature dependence of the NMR proton spin lattice relaxation rate. Solid lines are a fit assuming a direct instead of Raman-II process. The resulting calculated field dependence is clearly wrong since different fields do not overlap as in the data.

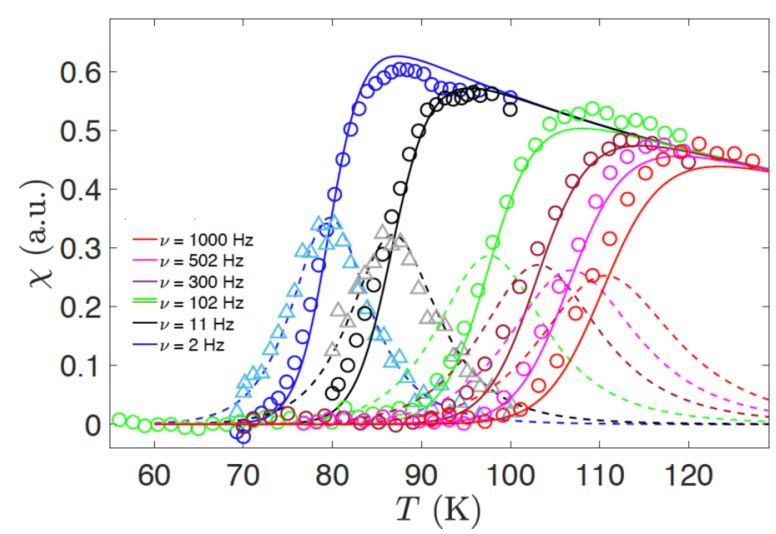

FIG. 8. Dynamic susceptibility $\chi(\omega)=\chi^{\prime}+i \chi^{\prime \prime}$ as a function of $T$. Due to the very weak signal, only the real part could be measured at high frequency (circles), while simulations are reported for both real (solid lines) and imaginary (dashed lines) parts. The position of the maximum of $\chi^{\prime \prime}$ coincides with the inflection point of $\chi^{\prime}$, indicating monoexponential relaxation.

modes are resonant at the energies corresponding to the $\mathrm{CF}$ gaps $\left(\Delta_{s t} \gtrsim 10 \mathrm{meV}\right)$, and thus, only these are involved in the Orbach process examined here. Diagonal elements are calculated as $W_{s s}=-\sum_{t} W_{t s}$, and the detailed balance condition $W_{s t} e^{-E_{t} / k_{B} T}=W_{t s} e^{-E_{s} / k_{B} T}$ is fulfilled. In 1 we find a single relaxation rate (orders of magnitude smaller than all the others) dominating the relaxation dynamics.

Interpretation of NMR measurements. The quasielastic spectrum of fluctuation of the system magnetization can be obtained [55,69] from the Fourier transform of the correlation function $\left\langle\Delta J_{z}(t) \Delta J_{z}(0)\right\rangle$, which consists of a weighted superposition of Lorentzians

$$
\mathcal{S}_{J_{z}, J_{z}}(\omega, T, B)=\sum_{i=1}^{2 J+1} A\left(\lambda_{i}, T, B\right) \frac{\lambda_{i}(T, B)}{\lambda_{i}(T, B)^{2}+\omega^{2}}
$$

centered at zero frequency and having a width provided by the eigenvalues $\lambda_{i}$ of $-\mathbb{W}$. The weights $A\left(\lambda_{i}, T, B\right)$ are calculated from the normalized eigenvectors of $\mathbb{W}$. It can be shown $[55,70]$ that the proton spin-lattice relaxation rate $1 / T_{1}$ detected by NMR measurements is proportional to $\mathcal{S}_{J_{z}, J_{z}}(\omega, T, B)$, with a coefficient $(\mathcal{A})$ accounting for the average position of the protons with respect to the magnetic ion:

$$
1 / T_{1}=\mathcal{A}^{2} \chi T \frac{\tau^{-1}}{\tau^{-2}+\omega_{L}^{2}},
$$

where $\omega_{L}=\gamma B(\gamma / 2 \pi \simeq 42 \mathrm{MHz} / \mathrm{T})$ is the proton Larmor frequency. In the temperature range examined here, $\tau^{-1} \ll$ $\omega_{L}$, and thus, $1 / T_{1} / \chi T \propto \mathcal{A}^{2} \tau^{-1} / B^{2}$. Measurements were collected at thermal equilibrium; thus, data shown in Fig. 3(b) have been divided by the equilibrium Curie susceptibility $\chi_{\mathrm{eq}}$ (see Fig. S15). Using our model from Eq. (4) for $\tau^{-1}$, we find the NMR data are reproduced extremely well with $\mathcal{A} \sim$ $1.5-1.7 \times 10^{8} \mathrm{rad} / \mathrm{s}$, which is compatible with that observed in other molecular systems with similar magnetic moment [71]. If a single rate dominates the dynamic, as observed in 1 in a wide frequency and temperature range, $\mathcal{S}_{J_{z}, J_{z}}$ is given by a single Lorentzian whose width corresponds to $\tau^{-1}$. 
[1] R. Sessoli, D. Gatteschi, A. Caneschi, and M. A. Novak, Nature (London) 365, 141 (1993).

[2] A. Chiesa, T. Guidi, S. Carretta, S. Ansbro, G. A. Timco, I. Vitorica-Yrezabal, E. Garlatti, G. Amoretti, R. E. P. Winpenny, and P. Santini, Phys. Rev. Lett. 119, 217202 (2017).

[3] S. T. Liddle and J. van Slageren, Chem. Soc. Rev. 44, 6655 (2015).

[4] J.-L. Liu, Y.-C. Chen, and M.-L. Tong, Chem. Soc. Rev. 47, 2431 (2018).

[5] A. Chiesa, F. Tacchino, M. Grossi, P. Santini, I. Tavernelli, D. Gerace, and S. Carretta, Nat. Phys. 15, 455 (2019).

[6] S. Thiele, F. Balestro, R. Ballou, S. Klyatskaya, M. Ruben, and W. Wernsodrfer, Science 344, 1135 (2014).

[7] A. Chiesa, P. Santini, D. Gerace, J. Raftery, A. A. Houck, and S. Carretta, Sci. Rep. 5, 16036 (2015).

[8] A. Chiesa, G. F. S. Whitehead, S. Carretta, L. Carthy, G. A. Timco, S. J. Teat, G. Amoretti, E. Pavarini, R. E. P. Winpenny, and P. Santini, Sci. Rep. 4, 7423 (2014).

[9] M. Shiddiq, D. Komijani, Y. Duan, A. Gaita-Ariño, E. Coronado, and S. Hill, Nature (London) 531, 348 (2016).

[10] J. Ferrando-Soria, E. Moreno-Pineda, A. Chiesa, A. Fernandez, S. A. Magee, S. Carretta, P. Santini, I. Victorica-Yrezabal, F. Tuna, E. J. L. McInness, and R. E. P. Winpenny, Nat. Commun. 7, 11377 (2016).

[11] J. Ferrando-Soria, S. A. Magee, A. Chiesa, S. Carretta, P. Santini, I. J. Vitorica-Yrezabal, F. Tuna, S. Sproules, K. M. Lancaster, A.-L. Barra, G. A. Timco, E. J. L. McInne, and R. E. P. Winpenny, Chem. 1, 727 (2016).

[12] R. Hussain, G. Allodi, A. Chiesa, E. Garlatti, D. Mitcov, A. Konstantatos, K. S. Pedersen, R. De Renzi, S. Piligkos, and S. Carretta, J. Am. Chem. Soc. 140, 9814 (2018).

[13] M. Atzori, A. Chiesa, E. Morra, M. Chiesa, L. Sorace, S. Carretta, and R. Sessoli, Chem. Sci. 9, 6183 (2018).

[14] A. Gaita-Ariño, F. Luis, S. Hill, and E. Coronado, Nat. Chem. 11, 301 (2019).

[15] Y. Furukawa, K. Kiuchi, K. Kumagai, Y. Ajiro, Y. Narumi, M. Iwaki, K. Kindo, A. Bianchi, S. Carretta, P. Santini, F. Borsa, G. A. Timco, and R. E. P. Winpenny, Phys. Rev. B 79, 134416 (2009).

[16] M. L. Baker, T. Lancaster, A. Chiesa, G. Amoretti, P. J. Baker, C. Barker, S. J. Blundell, S. Carretta, D. Collison, H. U. Gudel, T. Guidi, E. J. L. McInnes, J. S. Möller, H. Mutka, J. Ollivier, F. L. Pratt, P. Santini, F. Tuna, P. L. W. Tregenna-Piggott, I. J. Vitorica-Yrezabal, G. A. Timco, and R. E. P. Winpenny, Chem. Eur. J. 22, 1779 (2016).

[17] R. J. Woolfson, G. A. Timco, A. Chiesa, I. Vitorica-Yrezabal, F. Tuna, T. Guidi, E. Pavarini, P. Santini, S. Carretta, and R. E. P. Winpenny, Angew. Chem., Int. Ed. 128, 9002 (2016).

[18] G. A. Craig and M. Murrie, Chem. Soc. Rev. 44, 2135 (2015).

[19] J. M. Frost, K. L. M. Harriman, and M. Murugesu, Chem. Sci. 7, 2470 (2016).

[20] P. C. Bunting, M. Atanasov, E. Damgaard-Møller, M. Perfetti, I. Crassee, M. Orlita, J. Overgaard, J. van Slageren, F. Neese, and J. R. Long, Science 362, 1378 (2018).

[21] D. N. Woodruff, R. E. P. Winpenny, and R. A. Layfield, Chem. Rev. 113, 5110 (2013).

[22] S. Gómez-Coca, D. Aravena, R. Morales, and E. Ruiz, Coord. Chem. Rev. 289-290, 379 (2015).
[23] R. J. Blagg, L. Ungur, F. Tuna, J. Speak, P. Comar, D. Collison, W. Wernsdorfer. E. J. L. McInnes, L. Chibotaru, and R. E. P. Winpenny, Nat. Chem. 5, 673 (2013).

[24] S. Demir, M. I. Gonzales, L. E. Darago, W. J. Evans, and J. R. Long, Nat. Commun. 8, 2144 (2017).

[25] N. F. Chilton, C. A. P. Goodwin, D. P. Mills, and R. E. P. Winpenny, Chem. Commun. 51, 101 (2015).

[26] F. Pointillart, K. Bernot, S. Golhen, B. Le Guennic, T. Guizouarn, L. Ouahab, and O. Cador, Angew. Chem., Int. Ed. 54, 1504 (2015).

[27] Y.-S. Ding, N. F. Chilton, R. E. P. Winpenny, and Y.-Z. Zheng, Angew. Chem., Int. Ed. 55, 16071 (2016).

[28] J. Liu, Y.-C. Chen, J.-L. Liu, V. Vieru, L. Ungur, J.-H. Jia, L. F. Chibotaru, Y. Lan, W. Wernsdorfer, S. Gao, X.-M. Chen, and M.-L. Tong, J. Am. Chem. Soc. 138, 5441 (2016).

[29] Y.-C. Chen, J.-L. Liu, L. Ungur, J. Liu, Q.-W. Li, L.-F. Wang, Z.-P. Ni, L. F. Chibotaru, X.-M. Chen, and M.-L. Tong, J. Am. Chem. Soc. 138, 2829 (2016).

[30] S. K. Gupta, T. Rajeshkumar, G. Rajaraman, and R. Murugavel, Chem. Sci. 7, 5181 (2016).

[31] M. Gregson, N. F. Chilton, A.-M. Ariciu, F. Tuna, I. F. Crowe, W. Lewis, A. J. Blake, D. Collison, E. J. L. McInnes, R. E. P. Winpenny, and S. T. Liddle, Chem. Sci. 7, 155 (2016).

[32] C. A. P. Goodwin, F. Ortu, D. Reta, N. F. Chilton, and D. P. Mills, Nature (London) 548, 439 (2017).

[33] F.-S. Guo, B. M. Day, Y.-C. Chen, M.-L. Tong, A. Mansikkamäki, and R. A. Layfield, Science 362, 1400 (2018).

[34] K. R. McClain, C. A. Gould, K. Chakarawet, S. J. Teat, T. J. Groshens, J. R. Long, and B. G. Harvey, Chem. Sci. 9, 8492 (2018).

[35] Y.-S. Ding, K.-X. Yu, D. Reta, F. Ortu, R. E. P. Winpenny, Y.-Z. Zheng, and N. F. Chilton, Nat. Commun. 9, 3134 (2018).

[36] M. J. Giansiracusa, A. K. Kostopoulos, D. Collison, R. E. P. Winpenny, and N. F. Chilton, Chem. Commun. 55, 7025 (2019).

[37] A. Abragam and B. Bleaney, Electron Paramagnetic Resonance of Transition Metal Ions (Clarendon, Oxford, 1970).

[38] See Supplemental Material at http://link.aps.org/supplemental/ 10.1103/PhysRevB.101.174402 for details on synthesis, the model of magnetoelastic interaction, Raman integrals, INS, magnetization and NMR experiments, $a b$ initio calculation of the phonon DOS, the Brons-van Vleck contribution to Raman relaxation, hyperfine coupling, low-temperature quantum tunneling, and hysteresis loops, which includes Refs. [72-78].

[39] L. T. A. Ho and L. F. Chibotaru, Phys. Rev. B 97, 024427 (2018).

[40] M. N. Leuenberger and D. Loss, Phys. Rev. B 61, 1286 (2000).

[41] C. Calero, E. M. Chudnovsky, and D. A. Garanin, Phys. Rev. B 74, 094428 (2006).

[42] P. Thalmeier and B. Lüthi, Handbook on the Physics and Chemistry of Rare Earths, edited by K. A. Gschneider, Jr., and L. Eyring (Elsevier Science Publisher B. V. 1991), Vol. 14.

[43] K. N. Shrivastava, Phys. Status Solidi B 117, 437 (1983).

[44] The inclusion of a small dispersion for optical modes does not change the conclusions of the work.

[45] R. I. Bewley, R. S. Eccleston, K. A. McEwen, S. M. Hayden, M. T. Dove, S. M. Bennington, J. R. Treadgold, and R. L. S. Coleman, Physica B (Amsterdam, Neth.) 385-386, 1029 (2006). 
[46] A. Lunghi and S. Sanvito, Sci. Adv. 5, eaax7163 (2019).

[47] A. Albino, S. Benci, L. Tesi, M. Atzori, R. Torre, S. Sanvito, R. Sessoli, and A. Lunghi, Inorg. Chem. 58, 10260 (2019).

[48] R. Orbach, Proc. R. Soc. London, Ser. A 264, 485 (1961).

[49] R. Orbach, Proc. R. Soc. London, Ser. A 264, 458 (1961).

[50] J. H. Van Vleck, Phys. Rev. 57, 426 (1940).

[51] We assume internal fields have a Gaussian distribution with standard deviation $\sigma_{B}$ [38]; this behavior was recently observed in vanadyle molecular systems [79].

[52] D. C. Johnston, Phys. Rev. B 74, 184430 (2006).

[53] This value of $\sigma_{B}$ is smaller than that obtained in the intermediate-temperature regime because of the very different initial conditions. Indeed, low-temperature magnetization decay experiments are carried on by first saturating the sample in a large field, while the relaxation rates reported in Fig. 3(a) were obtained at higher temperatures and starting from $B=0$. The corresponding distribution of internal fields is expected to be much broader in the latter case, compared to the former [60], in agreement with our findings.

[54] The variation of $\tau^{-1}$ with $\theta$ strongly depends on the axiality of the ground doublet and on the form of the magnetoelastic coupling with acoustic phonons.

[55] P. Santini, S. Carretta, E. Liviotti, G. Amoretti, P. Carretta, M. Filibian, A. Lascialfari, and E. Micotti, Phys. Rev. Lett. 94, 077203 (2005).

[56] Experiments were performed at thermal equilibrium; hence, we can consider the equilibrium value for the Curie susceptibility in the expression of $1 / T_{1}$ (see Appendix D). Measurements performed below $20 \mathrm{~K}$ could deviate from thermal equilibrium and were thus excluded from our analysis.

[57] S. Gómez-Coca, A. Urtizberea, E. Cremades, P. J. Alonso, A. Camón, E. Ruiz, and F. Luis, Nat. Commun. 5, 4300 (2014).

[58] N. V. Prokof'ev and P. C. E. Stamp, Phys. Rev. Lett. 80, 5794 (1998).

[59] M. J. Martínez-Pérez, S. Cardona-Serra, C. Schlegel, F. Moro, P. J. Alonso, H. Prima-García, J. M. Clemente-Juan, M. Evangelisti, A. Gaita-Ariño, J. Sesé, J. van Slageren, E. Coronado, and F. Luis, Phys. Rev. Lett. 108, 247213 (2012).

[60] W. Wernsdorfer, T. Ohm, C. Sangregorio, R. Sessoli, D. Mailly, and C. Paulsen, Phys. Rev. Lett. 82, 3903 (1999).

[61] Here the internal transverse field (of the order of tens of milliteslas according to a rough estimation) is likely dominant in the low-field region in which tunneling is activated. Hence, we model the data with a Gaussian of constant amplitude $\Omega$, accounting for an average tunnel splitting.
[62] W. Wernsdorfer, S. Bhaduri, C. Boskovic, G. Christou, and D. N. Hendrickson, Phys. Rev. B 65, 180403(R) (2002).

[63] Indeed, the Bose-Einstein population factor decreases strongly with $\omega$, thus limiting the integral (3) to the low-frequency part. Furthermore, the assumption of nondispersive optical modes yields a factor $1 / \sqrt{\omega}$ in the couplings with optical phonons as opposed to the $\sqrt{\omega}$ term in the coupling with acoustic (Debye) phonons.

[64] G. A. Gehring and K. A. Gehring, Rep. Prog. Phys. 38, 1 (1975).

[65] V. Dohm and P. Fulde, Z. Phys. B 21, 369 (1975).

[66] A. Singh and K. N. Shrivastava, Phys. Status Solidi B 95, 273 (1979).

[67] C.-Y. Huang, Optical Phonons in Electron Spin Relaxation, Phys. Rev. 154, 215 (1967).

[68] S. Bertaina, B. Barbara, R. Giraud, B. Z. Malkin, M. V. Vanuynin, A. I. Pominov, A. L. Stolov, and A. M. Tkachuk, Phys. Rev. B 74, 184421 (2006).

[69] S. Carretta, P. Santini, G. Amoretti, M. Affronte, A. Candini, A. Ghirri, I. S. Tidmarsh, R. H. Laye, R. Shaw, and E. J. L. McInnes, Phys. Rev. Lett. 97, 207201 (2006).

[70] F. Adelnia, A. Chiesa, S. Bordignon, S. Carretta, A. Ghirri, A. Candini, C. Cervetti, M. Evangelisti, M. Affronte, I. Sheikin, R. Winpenny, G. Timco, F. Borsa, and A. Lascialfari, J. Chem. Phys. 143, 244321 (2015).

[71] Y. Furukawa, K. Watanabe, K. Kumagai, F. Borsa, T. Sasaki, N. Kobayashi, and D. Gatteschi, Phys. Rev. B 67, 064426 (2003).

[72] O. Arnold, J. C. Bilheux, J. M. Borreguero, A. Buts, S. I. Campbell, L. Chapon, M. Doucet, N. Draper, R. Ferraz Leal, M. A. Gigg, V. E. Lynch, A. Markvardsen, D. J. Mikkelson, R. L. Mikkelson, R. Miller, K. Palmen, P. Parker, G. Passos, T. G. Perring, P. F. Peterson, S. Ren, M. A. Reuter, A. T. Savici, J. W. Taylor, R. J. Taylor, R. Tolchenov, W. Zhou, and J. Zikovsky, Nucl. Instrum. Methods Phys. Res., Sect. A 764, 156 (2014).

[73] S. J. Clark, M. D. Segall, C. J. Pickard, P. J. Hasnip, M. J. Probert, K. Refson, and M. C. Payne, Zeitschrift für Kristallographie 220, 567 (2005).

[74] A. Togo and I. Tanaka, Scr. Mater. 108, 1 (2015).

[75] L. C. Hebel and C. P. Slichter, Phys. Rev. 113, 1504 (1959).

[76] G. Ablart and J. Pescia, Phys. Rev. B 22, 1150 (1980).

[77] A. Singh and R. C. Sapp, Phys. Rev. B 5, 1688 (1972).

[78] G. Allodi, A. Banderini, and R. De Renzi, Rev. Sci. Instrum. 76, 083911 (2005).

[79] M. Atzori, L. Tesi, E. Morra, M. Chiesa, L. Sorace, and R. Sessoli, J. Am. Chem. Soc. 138, 2154 (2016). 